\title{
Measuring Dynamic Interaction in Movement Data
}

Jed A. Long ${ }^{1}$, Trisalyn A. Nelson ${ }^{1}$

${ }^{1}$ Spatial Pattern Analysis \& Research (SPAR) Laboratory

Department of Geography, University of Victoria

*Corresponding author email \& address:

jlong@uvic.ca

Department of Geography, University of Victoria

PO Box 3060 STN CSC

Victoria, BC

V8W 3R4, Canada

\section{Pre-print of published version. \\ Reference: \\ Long, JA and TA Nelson. 2013. Measuring dynamic interaction in movement data. Transactions in GIS. 17(1). 62-77. \\ DOI: \\ http://dx.doi.org/10.1111/j.1467-9671.2012.01353.x \\ Disclaimer: \\ The PDF document is a copy of the final version of this manuscript that was subsequently accepted by the journal for publication. The paper has been through peer review, but it has not been subject to any additional copy-editing or journal specific formatting (so will look different from the final version of record, which may be accessed following the DOI above depending on your access situation).}

Running Head: Measuring Dynamic Interaction

Keywords: dynamic interaction, movement data, correlation, GPS, space-time, local statistics, spatial analysis 


\section{ABSTRACT:}

2 The emergence of technologies capable of storing detailed records of object locations has

3 presented scientists and researchers with a wealth of data on object movement. Yet

4 analytical methods for investigating more advanced research questions from such detailed

5 movement datasets remain limited in scope and sophistication. Recent advances in the

6 study of movement data has focused on characterizing types of dynamic interactions,

7 such as single-file motion, while little progress has been made on quantifying the degree

8 of such interactions. In this article, we introduce a new method for measuring dynamic

9 interactions (termed DI) between pairs of moving objects. Simulated movement datasets

10 are used to compare DI with an existing correlation statistic. Two applied examples, team

11 sports and wildlife, are used to further demonstrate the value of the DI approach. The DI

12 method is advantageous in that it measures interaction in both movement direction

13 (termed azimuth) and displacement. As well, the DI approach can be applied at local,

14 interval, episodal, and global levels of analysis. However the DI method is limited to

15 situations where movements of two objects are recorded at simultaneous points in time.

16 In conclusion, DI quantifies the level of dynamic interaction between two moving

17 objects, allowing for more thorough investigation of processes affecting interactive

18 moving objects. 


\section{Introduction}

21 The study of individual movement has entered a new era whereby researchers

22 from various fields can benefit from fine resolution object movement data. Technical

23 developments associated with location aware technologies, such as GPS, are transforming

24 representations of movement. Despite improvements in spatially explicit movement

25 datasets, the scope and sophistication of research questions are limited by a lack of

26 methods and analysis (Wolfer et al. 2001). Laube et al. (2007) suggest that within

27 geography, reliance of geographic information systems (GIS) and spatial statistics on 2-

28 dimensional representations may be limiting the development of more complex analyses

29 of movement, while disciplines outside of geography may be unaware of the power of

30 spatial (and space-time) analysis. To optimally utilize new movement datasets, analytical

31 techniques capable of addressing more advanced research questions are required.

32 Recently, the identification and measurement of dynamic interactions between

33 moving objects has become an active area of research, likely owing to readily available

34 fine granularity movement data. Dynamic interaction, a term from the wildlife ecology

35 literature, can be defined as the way the movements of two individuals are related

36 (Macdonald et al. 1980) or as inter-dependency in the movements of two individuals

37 (Doncaster 1990). Alternatively, the terms association (Stenhouse et al. 2005), relative

38 motion (Laube et al. 2005), and correlation (Shirabe 2006) have been used to refer to

39 dynamic interactions between moving objects in other examples. All of these terms refer

40 to the same general idea: identifying of how the movements of one individual are related

41 to another. Recent work on dynamic interactions has focused on methods for identifying

42 dynamic interaction patterns defined a priori (for example single file motion, Buchin et 
43 al. 2010; or chasing behavior, de Lucca Siqueira and Bogorny 2011). However limited

44 work exists on quantifying the strength of dynamic interactions present in movement

45 data. With this in mind we are motivated to investigate methods for measuring the

46 strength of dynamic interactions when there is an expectation that such behavior occurs.

47 This approach differs from recent developments in movement analysis which focus on

48 identifying patterns, defined a priori, from large movement databases.

49 The objective of this work is to extend a previously developed statistic (Shirabe

50 2006) to a measure capable of quantifying the degree of dynamic interaction between

51 moving objects. The new method (termed DI) measures dynamic interaction in

52 coincidental movement segments, that is, it requires movement data of two individuals

53 recorded simultaneously. The DI method is separable into components measuring

54 dynamic interaction in movement direction (azimuth) and movement distance

55 (displacement), termed $\mathbf{D} \mathbf{I}_{\theta}$ and $\mathbf{D} \mathbf{I}_{d}$ respectively. Further, $\mathbf{D I}$ is appropriate with the four

56 analysis levels (local, interval, episodal, and global - see Figure 1) identified by Laube et

57 al. (2007) with the beneficial property of local values (denoted here using lower-case -

58 di) that aggregate to the interval, episodal and global values. Lastly, DI is derived in a

59 way to allow for a time-lagged approach, but also extensions including time- and

60 distance-based weighting schemes.

$<$ Approximate location Figure 1 >

\section{$62 \quad 2$ Related Work}

63 This research is motivated by an existing technique (Shirabe 2006) for measuring

64 the strength of dynamic interactions (termed correlations) present in movement data. The

65 use of the term correlation by Shirabe stems from the fact that the statistic takes the form 
66 of a Pearson product-moment correlation coefficient. Consider two moving objects $M^{a}$

67 and $M^{b}$, whose spatial coordinates $(x, y)$ are recorded coincidentally at discrete times $t=1$

$68 \ldots n$, termed fixes. Now consider for any $M$ with $t=2 \ldots n, \boldsymbol{V}=\left[M_{t}-M_{t-1}\right]=\left[\boldsymbol{v}_{t}\right]$, is a

69 vector time series of $M$ with $n-1$ vector segments. A correlation statistic for movement

70 data defined this way takes the form (Shirabe 2006):

$71 \boldsymbol{r}\left(\boldsymbol{V}^{a}, \boldsymbol{V}^{b}\right)=\frac{\sum_{t=1}^{n-1}\left(\boldsymbol{v}_{t}^{a}-\overline{\boldsymbol{v}}^{a}\right) \cdot\left(\boldsymbol{v}_{t}^{b}-\overline{\boldsymbol{v}}^{b}\right)}{\sqrt{\sum_{t=1}^{n-1}\left|\boldsymbol{v}_{t}^{a}-\overline{\boldsymbol{v}}^{a}\right|^{2}} \sqrt{\sum_{t=1}^{n-1}\left|\boldsymbol{v}_{t}^{b}-\overline{\boldsymbol{v}}^{b}\right|^{2}}}$

72 Where $\overline{\boldsymbol{v}}=\frac{1}{n-1} \sum_{t=1}^{n-1} \boldsymbol{v}_{t}$ are mean coordinate vectors of $\boldsymbol{V}$. The correlation statistic $(\boldsymbol{r})$

73 is defined over the interval $[-1,1]$ with a score of 1 being perfect positive correlation and

74 a score of -1 perfect negative correlation, with 0 denoting no correlation.

75 The statistic $-\mathbf{r}$, could be advanced in three ways. First, it is dependant on the

76 mean vector of each path, and thus measures correlations in movement deviations from

77 their respective means. The statistic, r, cannot be used for testing direct interactions

78 between two moving objects unless their corresponding mean vectors are identical or

79 near identical. An improved statistic would not rely on this overall mean value. Second, $\mathbf{r}$

80 is unable to disentangle the effects of correlations in movement azimuth and distance,

81 while being sensitive to both. Decomposing such a statistic into components based on

82 movement direction (termed azimuth) and distance (displacement) would be beneficial,

83 as it would allow interactions in these two independent components of movement to be

84 analyzed separately. A third improvement would be a statistic that measures the

85 interaction of each individual movement segment (i.e., local level - Laube et al. 2007).

86 By definition, $\mathbf{r}$ produces a single resulting value for the entire path (i.e., global level - 
87 Laube et al. 2007). When movement patterns are characterized by periods of interactive

88 and non-interactive behavior, or varying levels of interactive behavior, a local level

89 statistic will allow a finer treatment of dynamic interactions.

90 Measurements of dynamic interaction in movement data have also been

91 developed by wildlife researchers interested in a finer understanding of wildlife

92 movement processes. The types of interactions studied in wildlife are classified as either

93 static or dynamic interactions (Doncaster 1990; Macdonald et al. 1980). Static interaction

94 relates to how two individuals use space coincidentally, while dynamic interaction

95 reflects how the movements of two individuals are related, for example attraction

96 (Macdonald et al. 1980). Typically, measures of dynamic interaction summarize the

97 proximity of simultaneous movement points. Doncaster (1990) introduced one such

98 measure of dynamic interaction based on the variance/covariance matrix of the spatial

99 coordinates of simultaneous wildlife telemetry fixes; others have used Euclidean distance

100 as an indicator of interaction (Bandeira de Melo et al. 2007; Stenhouse et al. 2005).

101 Stenhouse et al. (2005) further investigated dynamic interaction in grizzly bears (termed

102 associations) by measuring dynamic interaction in movement direction (azimuth $-\theta$ )

103 defined as:

$104 f_{t}\left(\theta_{t}^{a}, \theta_{t}^{b}\right)=\frac{\left|-\left(\theta_{t}^{a}-\theta_{t}^{b}\right)-180\right|}{180}$

105 Equation (2) ranges from $0-1$, with values of 1 when direction of movements is identical 106 and zero when completely opposite (i.e., at $180^{\circ}$ ).

107 Measuring dynamic interactions in moving object databases is also directly

108 related to a larger body of literature on identifying similar movement trajectories (Sinha

109 and Mark 2005; Vlachos et al. 2002; Yanagisawa et al. 2003). Similarity indices are 
110 commonly employed as a first-step for identifying broader patterns or for detecting

111 clusters in larger movement databases (Benkert et al. 2008; Gao et al. 2010). Moving

112 object pairs that are highly interactive could also be said to follow a similar trajectory in

113 many of these applications, and the methods for detecting dynamic interactions in

114 movement data could be useful for detecting similar movement trajectories.

115 Recently, many new techniques have been developed for categorizing various

116 dynamic interaction patterns commonly found in movement data. Laube et al. (2005)

117 developed a method for detecting RElative MOtion (REMO) classes based upon

118 interpreting patterns of movement direction in groups of moving objects. For example,

119 trend-setting, when one object moves with anticipation of the movement of others, is

120 identifiable using the REMO approach. Noyon et al. (2007) use changes in inter-object

121 distance and velocity to identify relative behavior such as collision avoidance. Benkert et

122 al. (2008) present an algorithm for finding flock patterns in movement databases; which

123 tests whether a group of moving objects are contained in a circle radius $r$ over a given

124 time interval. The study of flocking behavior is useful in the study of wildlife and crowd

125 dynamics (Batty et al. 2003). Buchin et al. (2010) have developed a method for

126 identifying single-file motion in groups of moving objects. Single-file motion is detected

127 using free-space diagrams, derived from the Fréchet distance metric for comparing

128 polygonal curves (Alt and Godau 1995). Related to single-file motion is the detection of

129 chasing behavior, identifiable using the algorithm proposed by de Lucca Siqueira and

130 Bogorny (2011). The methods mentioned above are capable of identifying specific types

131 of dynamic interactions in movement data as defined a priori. However, such methods 
132 are unable to quantify the strength of dynamic interactions present, thus motivating the

133 development of quantitative measures of dynamic interaction.

\section{Derivation}

135 In developing a measure of dynamic interaction we consider the rather optimal

136 data situation (as in Shirabe 2006) where two moving objects' ( $M^{a}$ and $M^{b}$ ) spatial

137 coordinates $(x, y)$ are recorded coincidentally at discrete times $t=1 \ldots n$, termed fixes.

138 For any $M$ with $t=2 \ldots n, V=\left[M_{t}-M_{t-1}\right]=\left[v_{t}\right]$, is a vector time series of $M$ with $n-1$

139 vector segments. For each movement segment define two fundamental properties:

140 direction $(\theta)$, termed azimuth, and length $(d)$, termed displacement. Azimuth $(\theta)$ is the

141 angle between a movement segment and a constant axis, most commonly the horizontal

142 axis (Figure 2a). Displacement $(d)$ is the Euclidean distance between two consecutive

143 fixes in a movement segment (Figure 2a). We are interested in deriving a measure of

144 dynamic interaction that separately quantifies interactions in azimuth and displacement

145 (Figure 2b-e).

1473.1 Azimuth $-\theta$

148 To investigate the interaction in movement azimuths we take the cosine of the 149 angle between them. This is simply calculated as:

$150 \quad \mathbf{d i}_{\theta}=f_{t}\left(\theta_{t}^{a}, \theta_{t}^{b}\right)=\cos \left(\theta_{t}^{a}-\theta_{t}^{b}\right)$

151 where $\theta_{t}$ is the angle of movement at time-step $t$. Here $f_{t}$ has a range of $[-1,1]$ as desired.

152 The function $\cos \left(\theta_{t}^{a}-\theta_{t}^{b}\right)$ is 1 when movement segments have the same orientation, 0

153 when movement segments are perpendicular, and -1 when in complete opposing

154 directions. In practice if either object (or both) do not move (3) is undefined, because $\theta_{t}$ is 
155 undefined. Thus, we must consider two alternative scenarios; first if one object moves

156 and one remains stationary, and second if both objects remain stationary. Here we make

157 the assumption that if one moves and the other remains stationary the two objects exhibit

158 no directional interaction, and if both are stationary they are positively interactive.

159 Considering these two alternative scenarios, a complete definition for (3) is:

160

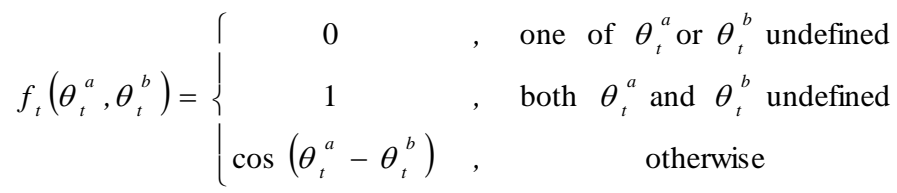

3.2 Displacement $-d$

162 Interaction in movement displacement could be measured using a variety of

163 functions. However, it is desirable to have the function $\left(g_{t}\right)$ fall in the range of $0-1$,

164 where a value of 0 represents no interaction and 1 positive interaction. Note there is no

165 consideration of negative interaction in displacement. Using this definition $g_{t}$ can be

166 thought of as a scaling function to $f_{t}$, and maintains the statistic on the range $[-1,1]$. We

167 propose the following function for $g_{t}$ :

$168 \quad \mathbf{d i}_{d}=g_{t}\left(d_{t}^{a}, d_{t}^{b}\right)=1-\left(\frac{\left|d_{t}^{a}-d_{t}^{b}\right|}{d_{t}^{a}+d_{t}^{b}}\right)^{\alpha}$

169 Where $|\cdot|$ is the absolute value operator, and $\alpha$ is a scaling parameter defaulting to 1 . The

170 function $g_{t}\left(d_{t}^{a}, d_{t}^{b}\right)$ approaches zero when $d_{t}^{a} \gg \gg d_{t}^{b}$ or vice-versa, and is 1 when $d_{t}^{a}=$

$171 d_{t}^{b}$. The effect of the scaling parameter $(\alpha)$ on the function $g_{t}\left(d_{t}^{a}, d_{t}^{b}\right)$ is demonstrated in

172 Figure 3. Parameter $\alpha$ can be adjusted to place stricter or looser requirements on

173 similarity in displacement denoting interaction. As $\alpha$ is increased larger differences in

174 displacement are still considered as positively interactive. A closer examination of (5) 
175 reveals that it is undefined when $d_{t}^{a}+d_{t}^{b}=0$, (i.e., both objects are stationary). If we

176 consider both objects remaining stationary as positive interaction, a more robust

177 definition of (5) is:

$178 \quad g_{t}\left(d_{t}^{a}, d_{t}^{b}\right)=\left\{\begin{array}{cc}1 & , \quad d_{t}^{a}+d_{t}^{b}=0 \\ 1-\left(\frac{\left|d_{t}^{a}-d_{t}^{b}\right|}{\mid d_{t}^{a}+d_{t}^{b}}\right)^{\alpha} & , \quad d_{t}^{a}+d_{t}^{b}>0\end{array}\right.$

180 Thus, for two corresponding movement segments, a measure of dynamic

181 interaction is the product between the azimuthal term $\left(f_{t}\right)$ and displacement term $\left(g_{t}\right)$ :

$182 \quad \mathbf{d i}{ }_{t}\left(v_{t}^{a}, v_{t}^{b}\right)=\mathbf{d i}{ }_{\theta} \times \mathbf{d i}_{d}=f_{t}\left(\theta_{t}^{a}, \theta_{t}^{b}\right) \times g_{t}\left(d_{t}^{a}, d_{t}^{b}\right)$

183 We are motivated to use the functions $f_{t}$ and $g_{t}$ to provide a statistic that covers the range

$184[-1,1]$ as was done in Shirabe (2006). Positive values of $\mathbf{d i}_{t}$ correspond to cohesive or

185 positively interactive movements, while negative values can be interpreted as repulsion or

186 opposing movements. Values near zero should be interpreted as having no interaction.

187 The di statistics measure dynamic interaction based on similarity in azimuth $(\theta)$

188 and displacement $(d)$ of simultaneous movement segments but do not account for the

189 proximity of moving objects. Thus, di represents a similarity index taken in a normalized

190 plane (i.e., the distance between the two objects has no impact on the resulting value).

191 We are motivated to use this type of formulation as the spatial proximity required for

192 dynamic interaction to occur is application specific. It is up to the analyst to decide if two

193 moving objects maintain a requisite proximity for dynamic interaction to occur, then such

194 interaction can be measured using di. In cases where actual spatial contact is required, for 
195 example when identifying points-of-interest in large movement databases (e.g., Benkert

196 et al. 2007), the di method should not be employed.

197 We have made assumptions in the equations for $\mathbf{d i}_{\theta}$ and $\mathbf{d i}_{\boldsymbol{d}}$ regarding how to

198 analyze dynamic interactions when objects do not move (i.e., $\theta$ is undefined and $d=0$ ).

199 In certain cases interpretation of these situations will be clear, for example, if one object

200 stops moving, does the other? However in practice, many applications may not facilitate

201 such straight-forward interpretation. For example, when studying urban travelers does

202 stopping at a red-light signify a change to dynamic interaction even if they will

203 eventually go straight? In light of these concerns, these assumptions can be modified to

204 accommodate different situations that may arise in various movement scenarios to fit a

205 given application.

2063.3 Global analysis

207 A global version of the di statistic can be used to measure the overall interaction 208 in a set of movement segments. First, it is useful to recognize that we can identify global

209 interaction in azimuth or displacement individually by summing the interaction values for

210 each individual segment and dividing by the number of segments. This form of a global

211 DI gives equal weight to each segment. .

212 DI ${ }_{\theta}\left(\boldsymbol{V}^{a}, \boldsymbol{V}^{b}\right)=\frac{1}{n-1} \sum_{t=1}^{n-1} \mathbf{d i}_{\theta}$

$213 \quad$ DI ${ }_{d}\left(V^{a}, V^{b}\right)=\frac{1}{n-1} \sum_{t=1}^{n-1} \mathbf{d i}_{d}$

214 A global measure of overall dynamic interaction DI can also be derived.

$$
\text { DI }\left(\boldsymbol{V}^{a}, \boldsymbol{V}^{b}\right)=\frac{1}{n-1} \sum_{t=1}^{n-1}\left(\mathbf{d i}_{\theta} \times \mathbf{d i}_{d}\right)=\frac{1}{n-1} \sum_{t=1}^{n-1}(\mathbf{d i})
$$


216 It is important to note that in the local version $\mathbf{d i}=\mathbf{d i}_{\theta} \times \mathbf{d i} \mathbf{i}_{d}$, but with the global statistic,

217 due to summation rules, $\mathbf{D I} \neq \mathbf{D I} \boldsymbol{\theta} \times \mathbf{D I}_{d}$. This can make interpretation of global values of

218 DI less straightforward than with local values. However, if we were to alternatively

219 define the global version as $\mathbf{D I}=\mathbf{D I}_{\boldsymbol{\theta}} \times \mathbf{D I}_{d}$, then the equation defined by (10) would no

220 longer hold. Thus, interpretation of DI values is best done separately for each component

221 (i.e., $\mathbf{D I}, \mathbf{D I}_{\boldsymbol{\theta}}$, and $\mathbf{D I} \mathbf{I}_{d}$ ).

222 The global formulation is also appropriate for interval and episodal levels of

223 analysis. Here we simply replace $n$ with some interval or episode length $n$ ', where $n$ ' $<n$.

224 This type of analysis can be illuminating when analyzing interactions in larger movement

225 datasets, where varying levels of dynamic interaction may occur at different points in the

226 movement paths.

227 3.4 Time- and Distance-based Weighting

228 In instances where the sampling interval of the $n$ fixes is unequal it is desirable to

229 scale the statistic based on the temporal duration of each movement segment. In practice,

230 this would give more weight to segments of longer duration and less weight to shorter

231 segments. Temporal weighting may also be used to account for missing fixes, common to

232 GPS-based tracking data. Let $\Delta_{t}$ correspond to the temporal duration of segment $t$, where

$233 \sum_{t=1}^{n-1} \Delta_{t}=T$ is the total duration of the entire movement path. Then a time weighted

234 version of (10) is defined as:

235 DI $\left(\boldsymbol{V}^{a}, \boldsymbol{V}^{b}\right)=\sum_{t=1}^{n-1} \frac{\Delta_{t}}{T} \mathbf{d i}_{\boldsymbol{\theta}} \times \mathbf{d i}_{d}$

236 Viewed in light of the uncertainty associated with movement data, this form of temporal

237 weighting may be counter-intuitive. That is, it may be logical to assign weights inversely 
238 proportional to the duration between fixes; lower weights to segments with higher

239 uncertainty (i.e., more time between fixes) and higher weights to segments with higher

240 certainty or finer space-time resolution.

241 Similarly, we can define a distance-based weighting scheme for (10) where

242 movements with larger displacement have increased weight in calculation of the statistic.

243 Varying distance-based weights could be used when dynamic interactions of a specific

244 movement behavior are of interest. For example in the study of wildlife long directed

245 movements are often interspersed with shorter random movements distinguishing

246 migratory and foraging behavior (Turchin 1998). Distance weighting could be used to

247 tailor the measurement of dynamic interactions to either of migratory or foraging

248 behaviors in this case. A possible distance-based weighting scheme would be the average

249 displacement of two segments: $d_{t}^{\text {avg }}=\left(d_{t}^{a}+d_{t}^{b}\right) / 2$, and $\sum_{t=1}^{n-1} d_{t}^{a v g}=D$. Based on the

250 average displacement a distance-weighted version of (10) is defined as:

$251 \quad$ DI $\left(\boldsymbol{V}^{a}, \boldsymbol{V}^{b}\right)=\sum_{t=1}^{n-1} \frac{d_{t}^{a v g}}{D} \mathbf{d i}_{\theta} \times \mathbf{d i}_{d}$

252 However, the average displacement of two objects movement segments is misleading

253 when one object has a large displacement and the other has a small displacement. Thus,

254 other distance measures are worth investigating for alternative distance-based weighting

255 schemes, keeping in mind that the sum of the weights should equal one. The equations

256 (11) and (12) can be combined to provide a time- and distance-based weighting scheme.

257 It is important to note that time- and distance-based weighting is really only useful when

258 interpreting global results when there is benefit to assigning segments weights based on

259 duration or distance. 
Another interesting extension to studying correlations in movement paths is when

261 movements interact with a temporal lag, for example when trend-setting occurs, as

262 described by Laube et al. (2005). The DI statistic can be modified to evaluate dynamic

263 interactions at a temporal lag. To measure dynamic interactions at a temporal lag, select a

264 time lag $-k$, where $k$ is generally taken to be a multiple of the fix interval (i.e., if fixes are

265 taken at even intervals the time between consecutive fixes). Then we can, alternatively

266 define $\mathbf{d i}_{\theta}$ and $\mathbf{d i} \mathbf{i}_{d}$ as:

$267 \quad \mathbf{d i}{ }_{\theta}=f_{t}\left(\theta_{t}^{a}, \theta_{t+k}^{b}\right)$

$268 \quad \mathbf{d i}_{d}=g_{t}\left(d_{t}^{a}, d_{t+k}^{b}\right)$

269 The global statistics (DI, $\mathbf{D I}, \mathbf{D I}_{\boldsymbol{d}}$ ) can be computed as before, using the time lagged

270 versions of $\mathbf{d i}_{\theta}(13)$ and $\mathbf{d i}_{\boldsymbol{d}}(14)$.

\section{Data}

272 4.1 Simulated Data

273 Six simulated data sets are used to highlight the utility of the DI statistic and the

274 benefit of extensions it makes to $\mathbf{r}$ (Shirabe 2006). A single random walk $(n=10)$ is used

275 to generate a movement path that is the bases for the simulation examples. We used

276 manual permutations to the spatial coordinates of the original random walk to produce 5

277 new movement paths that represent 5 unique dynamic interaction scenarios (Table 1).

278 The first scenario simulates two objects moving with strong-positive dynamic interaction.

279 The second scenario uses the same two paths as the first scenario, but one is rotated at

$28045^{\circ}$, simulating strong interaction in displacement, and low interaction in azimuth. The

281 third scenario simulates positive interaction in azimuth and no interaction in

282 displacement. The fourth scenario simulates negative interaction in azimuth and no 
283 interaction in displacement. The fifth scenario simulates no interaction in azimuth and

284 strong interaction in displacement. The sixth scenario uses a second independent random

285 walk to simulate random interactions between two moving objects.

286 < Approximate location of Table 1 >

2874.2 Athletes - Ultimate Frisbee

288 In team sports players (objects) movements are expected to be highly interactive.

289 Often a defending player is tasked with "covering" an offensive player, and their

290 movements are in reaction to that offensive player. In the sport of ultimate frisbee,

291 offensive players move about the field in an attempt to get open for a pass from their

292 teammates. Defending players cover them, in an attempt to intercept or dissuade passes

293 from being completed. As such, in ultimate frisbee the movements of an offensive player

294 and their defender are highly interactive. We used $5 \mathrm{~Hz}$ sports-specific GPS devices

295 (GPSports, Fyshwick, Australia) to monitor the movements of two ultimate frisbee

296 players over a one minute segment during a training game. In this example, the two

297 players cover each other for the entirety of the one minute period. A total of $n=276$ GPS

298 locations (out of a possible 300) were simultaneously recorded. Most of the missing

299 locations occur when the players are relatively stationary. At 5 GPS locations per second

300 this represents an extremely detailed movement dataset, appropriate for investigating the

301 intricate movements of athletes.

3024.3 Grizzly Bears in Alberta, Canada

303 To further demonstrate DI, we investigate a previously published dataset

304 containing GPS telemetry locations of a number of grizzly bears in Alberta, Canada

305 (Stenhouse et al. 2005). Stenhouse et al. (2005) revealed that various bear combinations 
306 showed evidence of dynamic interaction during different seasons, in particular male-

307 female interactions were strongest during spring when mating activity occurs. To

308 demonstrate DI, we examine one specific male-female bear combination that exhibited a

309 relatively strong association during the mating season (male (G006) and female (G010) -

310 see Fig. 4 in Stenhouse et al. 2005). Grizzly bear GPS collars were programmed to obtain

311 a location fix every four hours, however missing entries are frequent. As a result, only

312112 simultaneous GPS fixes were obtained for the two bears during period from May 28,

3132000 to July 08, 2000. In this example, we incorporate time-based weighting in order to

314 account for unevenness in fix intervals (ranging from 4 hours to over 6 days).

\section{$315 \quad 5$ Results}

\section{$316 \quad 5.1$ Simulated Data}

317 Using the six simulated datasets we compared global values for $\mathbf{D I}, \mathbf{D I} \mathbf{I}_{\boldsymbol{\theta}}$, and $\mathbf{D I}_{\boldsymbol{d}}$ 318 with Shirabe's (2006) r statistic (Figure 4) to reveal both the similarities and differences

319 between these two methods. In scenario 1, where both movements are highly interactive

320 in both displacement and azimuth, DI and $\mathbf{r}$ are very similar. In scenario 2 DI and $\mathbf{r}$ are

321 similar, however using the DI method we can identify that interaction is higher in

322 displacement $\left(\mathbf{D I}_{\boldsymbol{d}}=0.977\right)$, and lower in azimuth $\left(\mathbf{D I} \mathbf{I}_{\boldsymbol{\theta}}=0.664\right)$. In contrast, scenario 3

323 reveals a situation where DI and $\mathbf{r}$ exhibit substantially different results. Using $\mathbf{D I}_{\boldsymbol{\theta}}$ and

$324 \mathbf{D I}_{\boldsymbol{d}}$ we can further examine the nature of the interaction in both azimuth and

325 displacement, in this case $\mathbf{D} \mathbf{I}_{\boldsymbol{d}}=0.287$ and $\mathbf{D I} \mathbf{I}_{\boldsymbol{\theta}}=0.992$. High $\mathbf{D} \mathbf{I}_{\boldsymbol{\theta}}$ independent of $\mathbf{D I} \mathbf{I}_{\boldsymbol{d}}$

326 could be useful in measuring interactive movement patterns via different modes of

327 transportation (e.g., walking vs. biking), or scale independent movement behavior in

328 wildlife. Scenario 4 demonstrates an example where negative dynamic interaction is 
329 present (i.e., repulsion). In this case, $\mathbf{D I}$ is small and negative $(\mathbf{D I}=-0.278)$ due to low

330 interaction in displacement $\left(\mathbf{D I}_{\boldsymbol{d}}=0.280\right)$, while $\mathbf{r}_{\mathbf{x y}}$ is large and negative $(\mathbf{r}=-0.805)$.

331 Scenario 5, shows the case where low DI is a function of low interaction in azimuth (DI $\mathbf{I}_{\boldsymbol{\theta}}$

$332=-0.095)$, despite having a strong level of interaction in movement displacement $\left(\mathbf{D I} \mathbf{I}_{\boldsymbol{d}}=\right.$

333 0.979), while $\mathbf{r}_{\mathbf{x y}}=-0.532$. Measurement of high vs. low $\mathbf{D I} \mathbf{I}_{\boldsymbol{d}}$ independent of $\mathbf{D} \mathbf{I}_{\boldsymbol{\theta}}$ could be

334 used in behavior analysis to identify objects with similar diurnal activity patterns (i.e.,

335 temporal patterns of long and short movements). In Scenario 6, both DI and $\mathbf{r}$ show

336 values near 0 , as would be expected from two independent random motions. It is

337 interesting to note that $\mathbf{D I}_{\boldsymbol{d}}=0.649$ is relatively high in this example, as the random

338 walks used identical parameters for their displacement distributions.

339 < Approximate location of Figure $4>$

$340 \quad 5.2$ Athletes - Ultimate Frisbee

341 In the Ultimate Frisbee example, the two players positively interact in movement

342 azimuth $\left(\mathbf{D I}_{\theta}=0.682\right)$ and movement displacement $\left(\mathbf{D I}_{\boldsymbol{d}}=0.730\right)$. The global statistic

343 shows that a substantial level of interaction exists between the two athletes $(\mathbf{D I}=0.572)$.

344 Local analysis enables the identification of times/locations where the athletes exhibit

345 more or less interactive movements (Figure 5). In the ultimate frisbee example, local

346 analysis is more informative than the global measure, as the movement path consists of

347 many (shorter) movement segments. Maps of local di can be combined with a time-series

348 graph of $\mathbf{d i}, \mathbf{d i}{ }_{\theta}$, and $\mathbf{d i}_{\boldsymbol{d}}$ related to times/locations during the game where the defending

349 player did a poor job covering the offensive player. We use episodal level analysis to

350 segregate the movement paths into episodes of high vs. low interaction in order to further

351 investigate the interactive behavior of these two athletes. For example, from 0 - 20 and 38 
$352-40$ seconds (highlighted in blue in Figure 5), high and positive di values suggest the

353 defending player is providing good defensive coverage (for these two episodes $\mathbf{D I}=$

354 0.757). While from 20 - 38 seconds (highlighted in red in Figure 5) di values are much

355 lower, an indication of less interactive movement and poor defensive coverage (for this

356 episode $\mathbf{D I}=0.122$ ).

$<$ Approximate location of Figure $5>$

$358 \quad 5.3$ Grizzly Bears in Alberta, Canada

359 In the grizzly bear example it was revealed that the male and female bears showed

360 substantial interaction $(\mathbf{D I}=0.578)$ over the 42 day period from May 28, 2000 to July 8,

3612000 , using time-based weighting (see equation (11)) to account for missing fixes.

362 Similarly, time weighted results for azimuth $\left(\mathbf{D I}_{\theta}=0.663\right)$ and displacement $\left(\mathbf{D I}_{d}=\right.$

363 0.731) reveal that both azimuth and displacement were strongly related during this

364 period. Local analysis revealed that the strong interaction seen with the global results was

365 a function of highly cohesive movements during the middle of June, while at the

366 beginning of June the two animals show little interaction (see Figure 6). Again we

367 perform analysis at the episodal level for separate periods identified visually from the

368 local analysis as having low and high dynamic interaction (low interaction: May 28 -

369 June 09; high interaction: June 09-29). The period of high interactions has a time-

370 weighted $\mathbf{D I}=0.492$, while the period of low interaction has a time-weighted $\mathbf{D I}=0.029$.

371 Highly interactive behavior by mating grizzly bears is common in this region, as males

372 will attempt to confine female movements to a 'mating area' (Hamer and Herrero 1990).

373 Interpretation of maps and graphs of di facilitates the identification of where and when

374 such behavior occurs. 


\section{Discussion}

377 DI has three fundamental advantages over an existing method (Shirabe 2006) for

378 measuring interactions (termed correlations) in movement data. First, the existing method

379 follows a traditional correlation coefficient structure and is thus dependent on the mean

380 vector of a movement vector time series. In most cases, this mean movement vector will

381 have little relevance in the context of the analysis. However, in cases where interactions

382 are expected to occur relative to some mean movement trajectory, the method from

383 Shirabe (2006) is still advantageous. For instance, two objects moving radially from a

384 point (at some acute angle) may exhibit dynamic interaction (e.g., Fig. 4a in Shirabe

385 2006). Second, DI is explicitly decomposed into components measuring interaction in

386 movement azimuth and displacement. This property enables analysts to identify

387 situations where movements are related in one component but not the other. For example,

388 in scenario $3, \mathbf{D I}_{\boldsymbol{d}}$ is low, however strong interaction is present in $\mathbf{D I} \boldsymbol{\theta}$, indicating that the

389 objects move with similar azimuths but not displacements, a conclusion not discernable

390 from the $\mathbf{r}_{\mathbf{x y}}$ statistic. Lastly, the di statistics we have developed are calculated

391 independently for each simultaneous movement segment. The di values can be mapped

392 and analyzed in a time-series fashion providing a local level analysis. Local analysis

393 reveals spatial-temporal information about locations of increased or decreased interaction

394 along the movement trajectory. Furthermore, the local level statistics $\left(\mathbf{d i}, \mathbf{d i} \mathbf{i}_{\theta}\right.$, and $\left.\mathbf{d i}_{d}\right)$ are

395 easily aggregated to coarser levels of analysis (interval, episodal, and global).

396 Other research areas where measuring movement interactions could provide new

397 and unique insight include transportation, human-activity, and other wildlife and sporting 
398 examples. In transportation applications measuring interactions in large movement

399 databases could be used for generating information on commuter behavior. Examples

400 from human-activity research where interactions are important include tourist behavior

401 (e.g., Shoval and Isaacson 2007) or crowd dynamics (Batty et al. 2003). With wildlife

402 movement data, the detection of interactions is important in the study of resource

403 selection (Millspaugh et al. 1998) and social behavior (Bandeira de Melo et al. 2007;

404 Kenward et al. 1993), but also for examining offspring dependency, and inter-/intra-

405 species behavior. Finally, a number of sporting examples exist where measuring

406 movement interactions could provide new and unique insight including soccer, American

407 football, and ice hockey.

408 We use simulated movement data to highlight the advantages of DI over an

409 existing method in a small set of specific scenarios designed to show the range of

410 dynamic interactions present in movement data. When two movements are highly

411 interactive (e.g., scenario 1) both methods successfully identify the high level of dynamic

412 interaction. Also, when two movements show opposing or repulsive movements (e.g.,

413 scenario 4) both methods are able to identify this behavior. The value of the DI method is

414 demonstrated in scenarios 3, 4, and 5, where interactions in either azimuth or

415 displacement are coupled with no interaction in the other component. This type of

416 analysis may be useful, for example, when object movement is dependent on a temporal

417 factor. For instance, many wildlife species are active only at specific times of the day and

418 remain dormant during other periods. Measuring positive dynamic interactions in

419 displacement, irrespective of azimuth, may be useful in identifying whether or not 
420 different species or individuals operate with similar circadian cycles (Merrill and Mech

$4212003)$.

422 The example from athletes playing ultimate frisbee demonstrates the value of

423 measuring dynamic interactions at the local and episodal levels of analysis. Local and

424 episodal analysis revealed periods of varying degrees of dynamic interaction, which can

425 be related to player performance (i.e., how well the defensive player was able to cover the

426 offensive player). In many team sports, player evaluation has traditionally been

427 conducted by human observers. More recently, data driven analyses have become

428 common in the evaluation of players in team sports (e.g., Fearnhead and Taylor 2011).

429 When a player's movement can be directly related to specific abilities, for instance the

430 soccer example in Laube et al. (2005), the measurement of dynamic interactions, using

431 the DI method can enhance player evaluation using novel sport-specific movement

432 datasets.

433 The DI method we have developed requires that movement locations be recorded

434 simultaneously. Such a tidy form of movement data (i.e., where objects locations are

435 recorded simultaneously) may not always be available, limiting the ability to implement

436 this method. In such cases, path interpolation methods (e.g., Tremblay et al. 2006) could

437 be used to estimate the locations of one object at coinciding times. Similarly, in many

438 applications the assumption that movement data are collected at a regular interval is not

439 satisfied (e.g., with movement data collected using cell-phone records). This is also the

440 case in many wildlife telemetry studies where missing fixes are common. In the grizzly

441 bear example, we demonstrate the value of temporal weighting the DI statistic to account

442 for uneven sampling intervals. Further, we highlighted how local and episodal analyses 
443 can provide unique and valuable insights into the nature of dynamic interactions present

444 in movement datasets. Local analysis reveals the times and locations of dynamic

445 interactions not discernable from global level statistics. When comparing male and

446 female grizzly bears, the dynamic interactions were likely due to mating behavior. This

447 example demonstrates the value of quantifying dynamic interactions in wildlife

448 movement datasets, as they can be related directly to specific social activities.

449 When movement data are collected at too fine a granularity, the movement

450 process (e.g., dynamic interaction) can be masked by data noise (termed over-sampling,

451 Turchin 1998). In these cases, down-sampling can be used to reduce data redundancy in

452 the movement path and improve the process signal to noise ratio. The DI statistics can

453 then be computed on the re-sampled movement dataset, as another form of interval and/or

454 episodal analysis (e.g., Laube et al. 2007). Variations of this procedure at different

455 interval and episodal scales can lead to increasingly complex and cross-scale

456 investigations of dynamic interactions in moving object datasets. Recently, Laube and

457 Purves (2011) have discussed the impact that movement data granularity (i.e., sampling

458 resolution) has on metrics used to quantify and describe movement trajectories (e.g.,

459 mean speed). The DI method is similarly impacted by the granularity at which

460 movement data are represented. For example, at a coarse granularity objects may exhibit

461 positive dynamic interactions, while at a fine granularity their movements may show

462 negative dynamic interaction (see Figure 7). Both the granularity at which the data are

463 represented and analysis level selected will impact the results and subsequent

464 interpretation of DI. One of Laube and Purves (2011) main recommendations is that 
465 movement data analysis be conducted across a range of scales (granularities and analysis

466 levels) to correctly understand observed patterns.

\section{Conclusions}

469 Movement data are being collected for a variety of research agendas involving the 470 study of humans, their vehicles, and wildlife. Central to analyzing movement data is the 471 measurement of dynamic interactions between pairs of moving objects. We have

472 developed a new statistic (DI) for measuring dynamic interactions in discrete movement

473 data (e.g., with a GPS). The basic properties of movement segments - azimuth and

474 displacement, are used to detect dynamic interactions in azimuth, displacement, and

475 overall movement. The DI method can be applied at four analysis levels (local, interval, 476 episodal, and global - Laube et al. 2007) associated with movement data, and results can

477 be aggregated across analysis levels. We introduce both time- and distance-based

478 weighting schemes that can be useful in specific situations. The measurement of dynamic 479 interactions at a temporal-lag, an example of trend-setting (Laube et al. 2005), can be 480 easily incorporated. Like many spatial analysis techniques the DI method is impacted by 481 the granularity at which movement data is represented. A detailed investigation of cross482 scale effects is warranted to provide a better understanding of how the measurement of 483 dynamic interaction is impacted by changing data granularities.

484 In some situations the nature of movement interactions will not simply involve 485 two moving objects, but rather involve two moving objects impacted by a third. Consider 486 the grizzly bear example; the bears exhibit varying levels of dynamic interaction over the 487 course of the time period. The level of interaction is likely affected by their position 
488 relative to the location of other objects, including other bears, roads, or sources of

489 attraction or repulsion (i.e., food or danger). Future research will develop approaches for

490 measuring third-party interactions, whereby pairs of moving objects interact with respect

491 to a third stationary or moving object.

492 To those wishing to measure dynamic interactions with their own applications we

493 have developed code for implementing DI in the statistical software package R ( R

494 Development Core Team 2011), for more information please visit:

495 <insert link to website here>

496 Acknowledgements

497 Funding for this work was provided by Canada's Natural Science and

498 Engineering Research Council (NSERC) and GEOIDE through the Government of

499 Canada's Networks for Centres of Excellence program. A special thanks to G. Stenhouse

500 and the Foothills Research Institute for access to the grizzly bear data used in the case

501 study. Also, thanks go out to the University of Victoria Ultimate Frisbee Club for their

502 participation in ongoing data collection endeavors. The constructive comments we

503 received from three anonymous reviewers greatly improved the presentation of this

504 article.

505 
506

507

508

509

510

511

512

513

514

515

516

517

518

519

520

521

522

523

524

525

526

527

528

529

530

531

532

533

534

535

536

537

538

539

540

541

542

543

544

545

546

547

548

549

550

\section{References}

Alt H and Godau M 1995 Computing the Fréchet distance between two polygonal curves. International Journal of Computational Geometry \& Applications 5 (1-2): 75-91

Bandeira de Melo L F, Lima Sabato M A, Vaz Magni E M, Young R J and Coelho C M 2007 Secret lives of maned wolves (Chrysocyon brachyurus Illiger 1815): as revealed by GPS tracking collars. Journal of Zoology 271 27-36

Batty M, Desyllas J and Duxbury E 2003 The discrete dynamics of small-scale events: agent-based models of mobility in carnivals and street parades. International Journal of Geographical Information Science 17 (7): 673-97

Benkert M, Djordjevic B, Gudmundsson J and Wolle T 2007 Finding popular places. In Tokuyama T (eds) Proceedings of the ISAAC 2007, LNCS 4835: 776-87

Benkert M, Gudmundsson J, Hubner F and Wolle T 2008 Reporting flock patterns. Computational Geometry 41 111-25

Buchin K, Buchin M and Gudmundsson J 2010 Constrained free space diagrams: a tool for trajectory analysis. International Journal of Geographical Information Science 24 (7): 1101-25

de Lucca Siqueira F and Bogorny V 2011 Discovering chasing behavior in moving object trajectories. Transactions in GIS 15 (5): 667-88

Doncaster C P 1990 Non-parametric estimates of interactions from radio-tracking data. Journal of Theoretical Biology 143 431-43

Fearnhead P and Taylor B M 2011 On estimating the ability of NBA players. Journal of Quantitative Analysis in Sports 7 (3): Article 11

Gao Y, Zheng B, Chen G and Li Q 2010 Algorithms for constrained $k$-nearest neighbor queries over moving object trajectories. Geoinformatica 14 241-76

Hamer D and Herrero S 1990 Courtship and use of mating areas by grizzly bears in the front ranges of Banff National Park, Alberta. Canadian Journal of Zoology 68 2695-97

Kenward R E, Marcstrom V and Karlbom M 1993 Post-nestling behaviour in goshawks, Accipiter gentilis: II. Sex differences in sociality and nest-switching. Animal Behaviour 46 371-78

Laube P, Imfeld S and Weibel R 2005 Discovering relative motion patterns in groups of moving point objects. International Journal of Geographical Information Science 19 (6): 639-68

Laube P, Dennis T, Forer P and Walker M 2007 Movement beyond the snapshot Dynamic analysis of geospatial lifelines. Computers, Environment and Urban Systems 31 481-501

Laube P and Purves R S 2011 How fast is a cow? Cross-scale analysis of movement data. Transactions in GIS 15 (3): 401-18

Macdonald D W, Ball F G and Hough N G 1980 The evaluation of home range size and configuration using radio tracking data. In Amlaner $\mathrm{C} \mathrm{J}$ and Macdonald $\mathrm{D} \mathrm{W}$ (eds) A Handbook on Biotelemetry and Radio Tracking. Oxford, Pergamon Press: 405-24

Merrill S B and Mech L D 2003 The usefulness of GPS telemetry to study wolf circadian and social activity. Wildlife Society Bulletin 31 (4): 947-60 
Millspaugh J J, Skalski J R, Kernohan B J, Raedeke K J, Brundige G C and Cooper A B 1998 Some comments on spatial independence in studies of resource selection. Wildlife Society Bulletin 26 (2): 232-36

Noyon V, Claramunt C and Devogele T 2007 A relative representation of trajectories in geographical spaces. Geoinformatica 11 479-96

R Development Core Team 2011 R: A language and environment for statistical computing. R Foundation for Statistical Computing. ISBN 3-900051-07-0, URL http://www.R-project.org Vienna, Austria

Shirabe T 2006 Correlation analysis of discrete motions. In Raubal M, Miller H J, Frank A U and Goodchild M F (eds) GIScience 2006. LNCS, vol. 4197. Berlin, Springer-Verlag: 370-82

Shoval N and Isaacson M 2007 Sequence alignment as a method for human activity analysis in space and time. Annals of the Association of American Geographers 97 (2): 282-97

Sinha G and Mark D M 2005 Measuring similarity between geospatial lifelines in studies of environmental health. Journal of Geographical Systems 7 115-36

Stenhouse G B, Boulanger J, Lee J, Graham K, Duval J and Cranston J 2005 Grizzly bear associations along the eastern slopes of Alberta. Ursus 16 (1): 31-40

Tremblay Y, Shaffer S A, Fowler S L, Kuhn C E, McDonald B I, Weise M J, Bost C A, Weimerskirch H, Crocker D E, Goebel M E and Costa D P 2006 Interpolation of animal tracking data in a fluid environment. The Journal of Experimental Biology 209 128-40

Turchin P 1998 Quantitative Analysis of Movement: Measuring and Modelling Population Redistribution in Animals and Plants. Sunderland, MA, Sinauer

Vlachos M, Gunopulos D and Kollios G 2002 Robust similarity measures for mobile object trajectories. In (eds) Proceedings of the 5th International Workshop on Mobility in Databases and Distributed Systems (MDDS), Aix-en-Provence, France: 721-26

Wolfer D P, Madani R, Valenti P and Lipp H-P 2001 Extended analysis of path data from mutant mice using the public domain software Wintrack. Physiology \& Behavior $73745-53$

Yanagisawa Y, Akahani J I and Satoh T 2003 Shape-based similarity query for trajectory of mobile objects. In Chen M-S, Chysanthis P K, Sloman M and Zaslavsky A (eds) Mobile Data Management, LNCS 2574. Berlin, Springer-Verlag: 63-77 
Table 1: Simulated movement scenarios, depicting different types of dynamic interactions, used to examine the differences between the new interaction statistic (DI) and an existing method (r).

\begin{tabular}{|c|l|l|}
\hline Scenario & Azimuth $(\boldsymbol{\theta})$ & Displacement $(\boldsymbol{d})$ \\
\hline $\mathbf{1}$ & Positive interaction & Interaction \\
\hline $\mathbf{2}$ & $\begin{array}{l}\text { Positive interaction } \\
\left(\text { rotated by } 45^{\circ}\right)\end{array}$ & Interaction \\
\hline $\mathbf{3}$ & Positive interaction & No interaction \\
\hline $\mathbf{4}$ & Negative interaction & No interaction \\
\hline $\mathbf{5}$ & No interaction & Interaction \\
\hline $\mathbf{6}$ & Random & Random \\
\hline
\end{tabular}




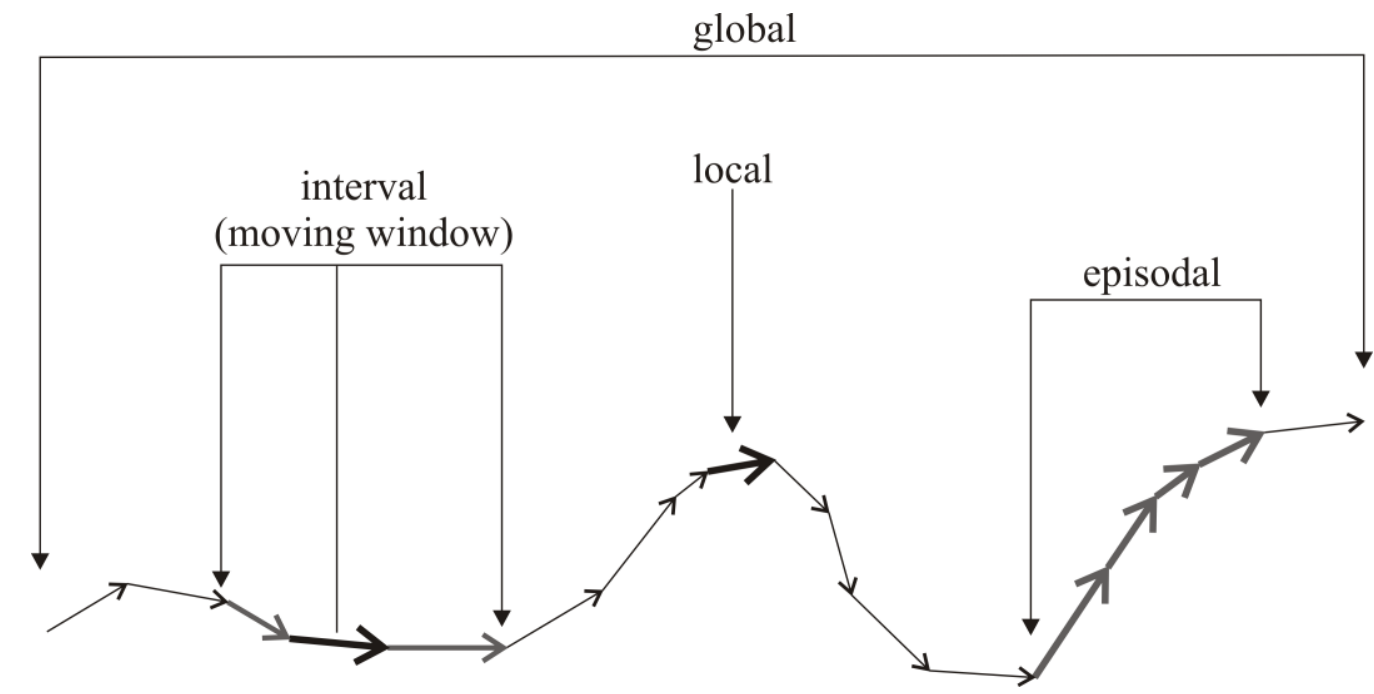

Figure 1: Diagram of four analysis levels used in movement data analysis (after Figure 2 in Laube et al. 2007). Local level statistics are calculated for each individual movement segment. Interval level analysis computes a running average statistic using a moving window. Episodal level analysis computes the statistic over a selected 'episode' or period of the dataset. Global level analysis computes the statistic over the entire movement path. 
a)

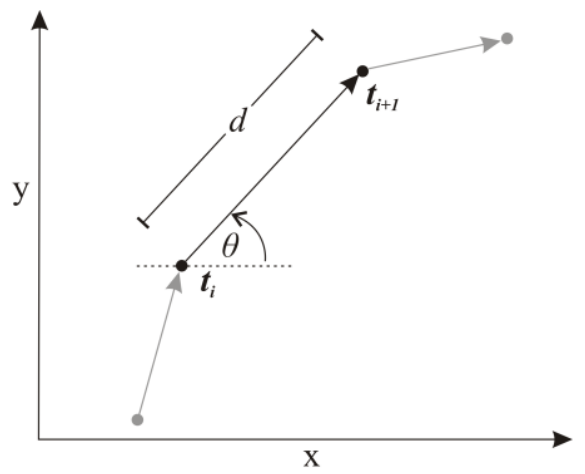

b)

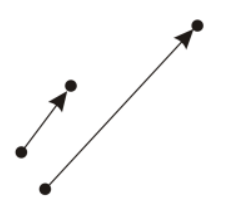

d)

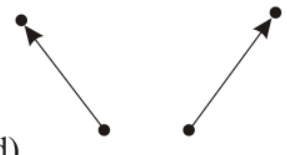

c)

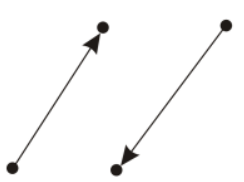

e)

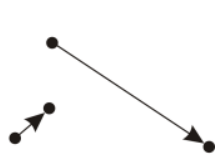

Figure 2: a) Diagram of movement properties azimuth $(\theta)$ and displacement $(d)$. Examples of movement segments that exhibit: b) positive interaction in $\theta$ and low interaction in $d$; c) negative interaction in $\theta$ and high interaction in $d$; d) no interaction in $\theta$ and high interaction in $d$; and e) no interaction in $\theta$ or $d$. 

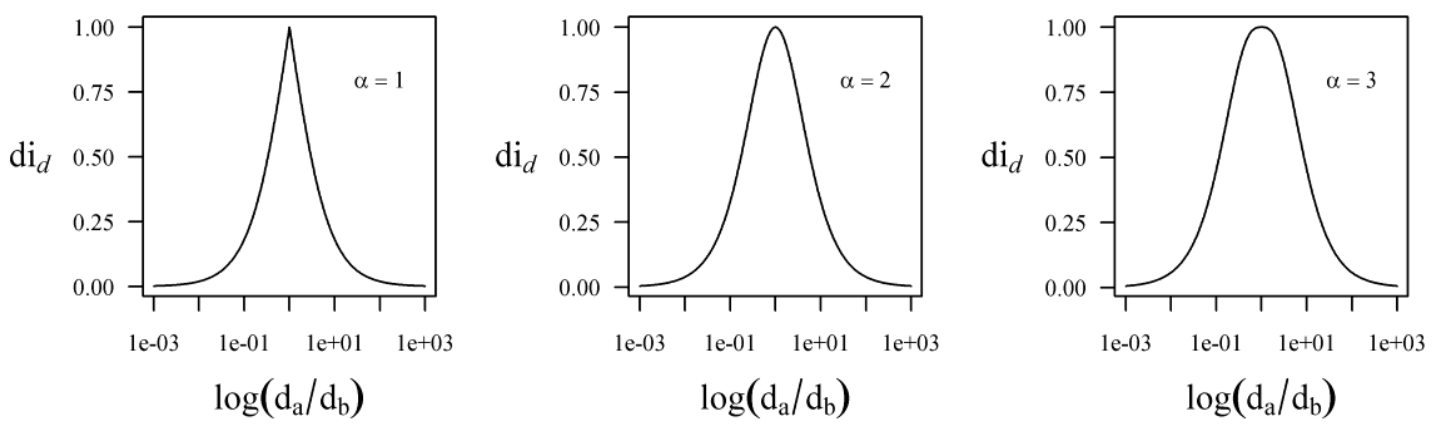

Figure 3: Relationship between $\log \left(d_{a} / d_{b}\right)$ and $\mathbf{d i}_{d}$, for values of $\alpha=1,2,3$. 


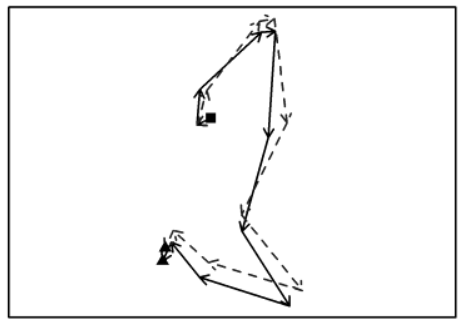

1) $\mathbf{D I}=0.969$

$\mathbf{D I}_{\theta}=0.991$

$\mathbf{D I}_{d}=0.979$

$\mathbf{r}=0.889$

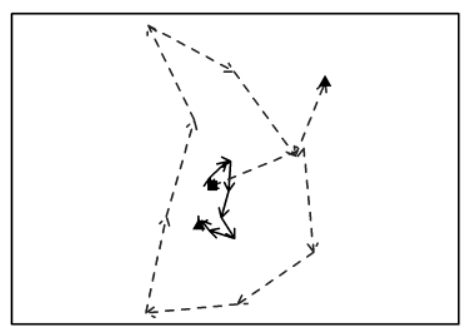

4) $\mathbf{D I}=-0.278$

$\mathbf{D I}_{\theta}=-0.994$

$\mathbf{D I}_{d}=0.280$

$\mathbf{r}=-0.805$

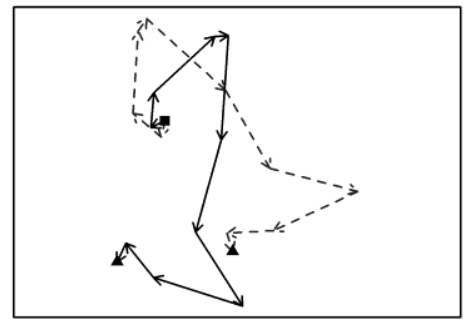

2) $\mathbf{D I}=0.650$

$\mathbf{D I}_{\theta}=0.664$

$\mathbf{D I}_{d}=0.977$

$\mathbf{r}=0.600$

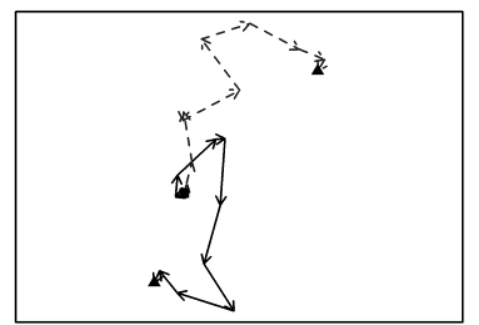

5 ) $\mathbf{D I}=-0.091$

$\mathbf{D I}_{\theta}=-0.095$

$\mathbf{D I}_{d}=0.979$

$\mathbf{r}=-0.532$

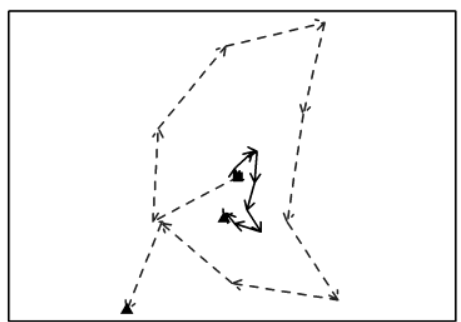

3 ) $\mathbf{D I}=0.285$

$\mathbf{D I}_{\theta}=0.992$

$\mathbf{D I}_{d}=0.287$

$\mathbf{r}=0.760$

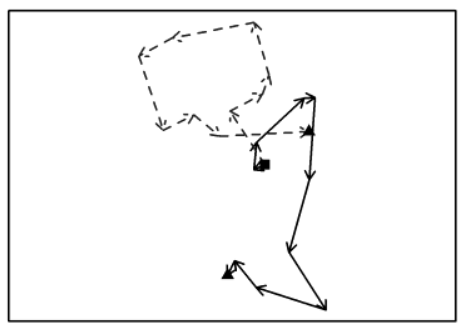

6) $\mathbf{D I}=0.091$

$\mathbf{D I}_{\theta}=0.112$

$\mathbf{D I}_{d}=0.649$

$\mathbf{r}=0.172$

Figure 4: Results from global analysis of 6 simulated example scenarios, comparing the new DI method with the Shirabe (2006) correlation statistic - r. Original path is solid and black, while the path in dashed grey portrays variations based on six simulated scenarios (see Table 1). 


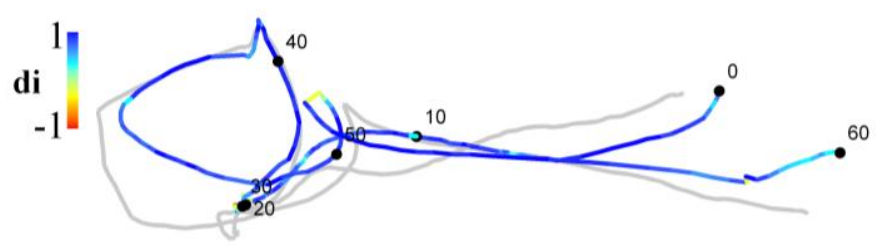

a) di map for player 1 (player 2 in gray)

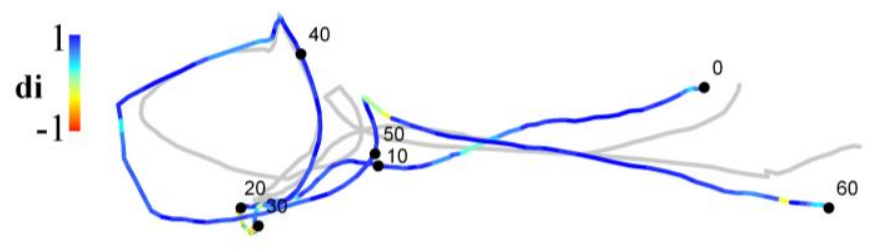

b) di map for player 2 (player 1 in gray)
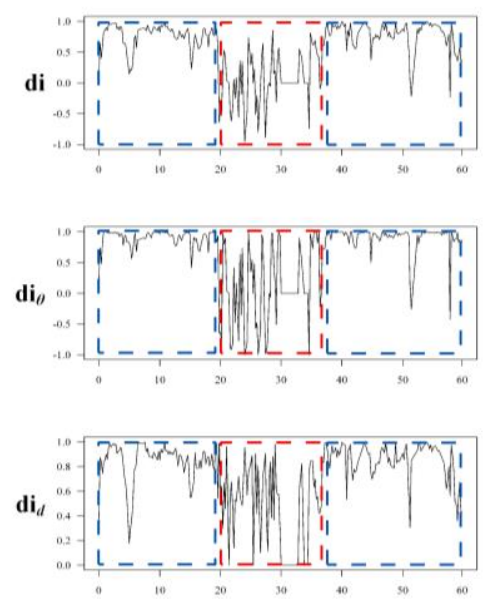

c) time series graphs of $\mathbf{d i}, \mathbf{d i}_{\theta}$ and $\mathbf{d i}_{d}$

Figure 5: Local analysis showing maps of di values for a) player 1, and b) player 2, from the ultimate frisbee example. c) time series graphs of $\mathbf{d i}, \mathbf{d i} \mathbf{i}_{\theta}$, and $\mathbf{d i}_{\boldsymbol{d}}$ can be used to identify periods of high and low dynamic interaction. Highlighted in blue in the time series graphs (c) are periods where player 1 does a good job covering player 2 (DI = 0.757). Highlighted in red is a period where the player 1 does a poorer job covering player $2(\mathbf{D I}=0.122)$. 


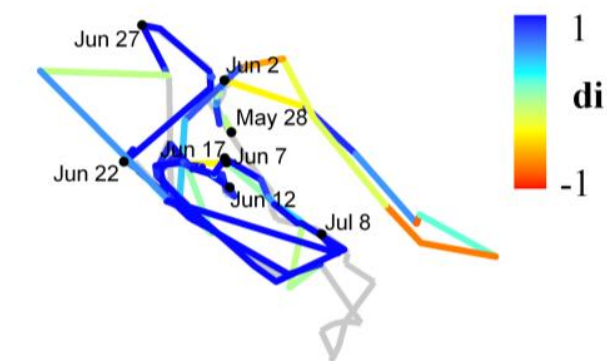

a) di map of male grizzly bear (female bear in gray)

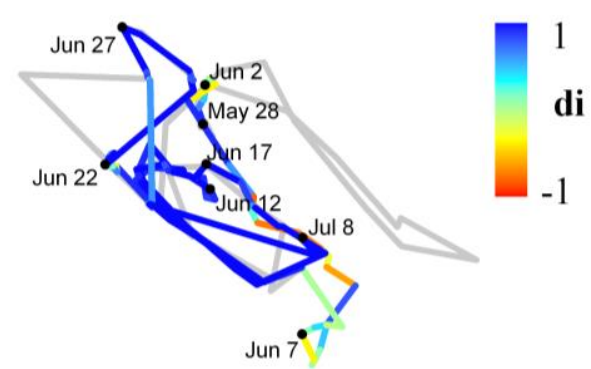

b) di map of female grizzly bear (male bear in gray)
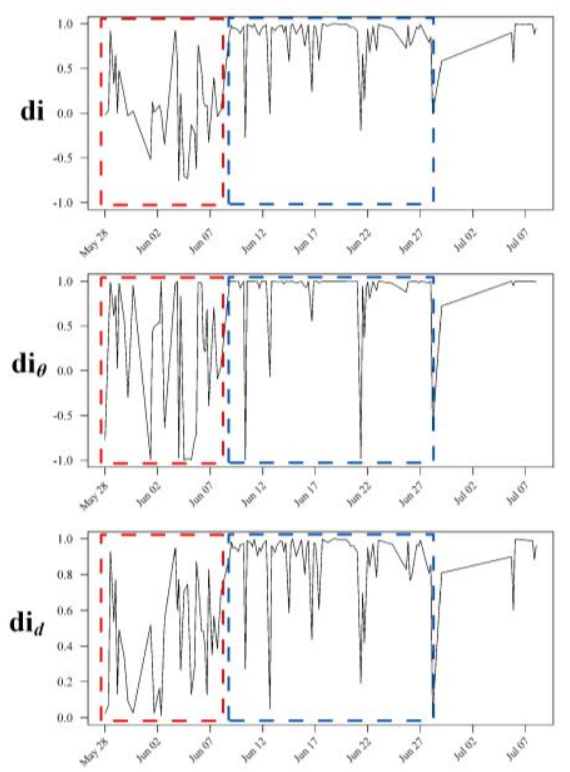

c) time series graphs of $\mathbf{d i}, \mathbf{d i}_{\theta}$, and $\mathbf{d i}_{d}$

Figure 6: Local analysis showing maps of di values for a) the male grizzly bear (G006), and b) the female grizzly bear (G010), from the grizzly bear example. c) time series graphs of $\mathbf{d i}, \mathbf{d i}_{\theta}$, and $\mathbf{d i}_{d}$ can be used to identify periods of high and low dynamic interaction. Highlighted in red in the time series graphs (c) is a period where the bears exhibit low dynamic interaction ( $\mathbf{D I}=0.029$ ). Highlighted in blue is period where the bears exhibit strong dynamic interaction $(\mathbf{D I}=0.492)$, in this example indicative of mating behavior. 

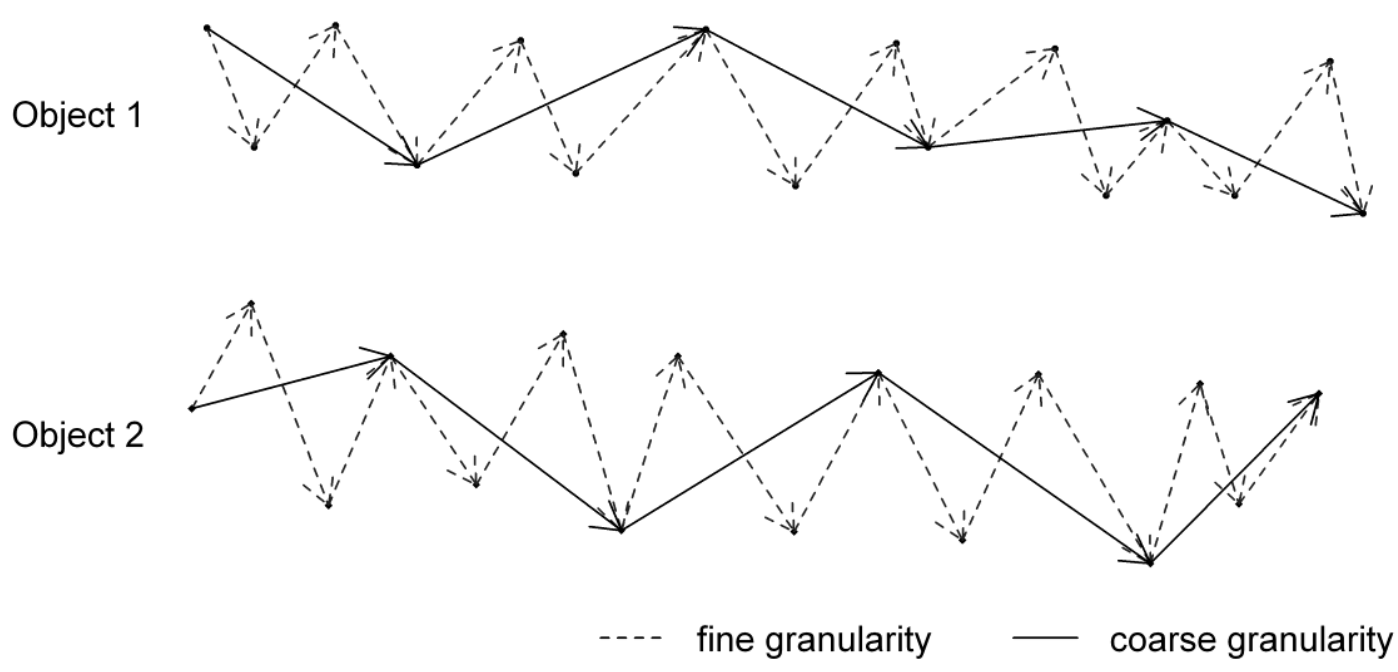

Figure 7: A pair of moving objects that exhibit negative dynamic interaction when analyzed at a fine granularity (dashed line, $\mathbf{D I}=-0.47$ ) but positive dynamic interaction when analyzed at a coarser granularity (solid line, $\mathbf{D I}=0.49$ ). This example illustrates how changes in data granularity can impact results and interpretation of DI. 